\title{
Anchor utilization trends with the implementation of a triple-loaded rotator cuff anchor
}

\begin{abstract}
As rotator cuff procedures have moved from open to arthroscopic, more attention has been paid to the use of anchors due to cost concerns and utilization of the real estate of the greater tuberosity footprint. A retrospective case series was performed to analyze anchor utilization during arthroscopic rotator cuff repair after triple-loaded anchors were available in comparison to the use of double-loaded anchors. One consecutive group of 69 patients had RCR with double-loaded anchors and a second consecutive group of 77 patients had RCR after triple-loaded anchors were available. For RC tear size greater than $2.5 \mathrm{~cm}^{2}$ the use of triple-loaded anchors resulted in a decreased use of nearly 1 anchor per repair. Level of evidence IV.
\end{abstract}

Keywords: rotator cuff, arthroscopy, triple-loaded

Volume II Issue 4 - 2019

\author{
Blair A Rhode, William S Rhode \\ Orland Park Orthopedics, USA
}

Correspondence: Blair A Rhode, Orland Park Orthopedics, I6450 S 104th AV, Orland Park, Illinois 60467, USA, Tel 708-364844I, Fax 708-364-8443, Email Blairbones@gmail.com

Received: August 28, 2019 | Published: August 30, 2019
Abbreviations: RC, rotator cuff; RCR, rotator cuff repair; ARCR, arthroscopic rotator cuff repairs; A-P, anterior-posterior; M-L, medial-lateral; StDev, standard deviation; DL, double-load; TL, triple-load; UHMWPE, ultrahigh molecular weight polyethelene; RöG, Rhode orthopedic group; PEEK, polyetheretherketone

\section{Introduction}

Arthroscopic rotator cuff repair (ARCR) surgery has grown in popularity as the subjective and functional outcome has improved over time. National trends in rotator cuff repair have demonstrated a $600 \%$ increase in arthroscopic procedures from 1996-2006. ${ }^{1}$ There are multiple repair techniques, each with their own purported benefits. The most commonly evaluated repair techniques are the single row and double row repair. Whether single-row (SR) or double-row (DR) result in a clinically significant difference has not been fully resolved. ${ }^{2-4}$ Advancements in anchor technology have focused on material usage or fixation methods. The development of a triple loaded anchor (3 suture limbs) has the potential advantage of decreasing anchor usage. This has the advantage of saving greater tuberosity real estate and decreasing repair costs. The average selling price (ASP) for a shoulder anchor was \$389 in 2017 Q3 (Orthopedic Network News, Vol 29(1) 2018).

A number of studies conducted using triple-loaded anchors concluded that SR repair was as good or even superior to DR repair. ${ }^{5-7}$ When double-row suture-bridge repair was compared to single-row repair using triple loaded anchors for $1-3 \mathrm{~cm}$ rotator cuff tears (RCTs), there was similar improvement in pain, function, and healing. ${ }^{8-10}$ A recent report in 2016 of anchor usage for RCRs at the Cleveland Clinic performed by 13 surgeons in 925 cases $^{11}$ provides a comparison their results to those of the present study.

It is our belief that the use of triple-loaded anchors improves footprint restoration by an increase of the number of suture passes through the ruptured tendon which has been shown to increase ultimate failure load. The number of anchors that can be safely implanted is constrained by the limited humeral head area. Triple-loaded anchors allow more passes through the tendon with a reduction in the number of anchors used in a repair.
The purpose of this study of the utilization rate of triple-load versus double-load anchors was to evaluate rotator cuff anchor utilization before and after the triple loaded anchor became part of the surgeon's implant choice.

\section{Methods}

This is a retrospective Level 3 Case series of two groups of patients that underwent arthroscopic rotator cuff repairs (ARCRs). 69 patients (48 male/21 female) average age was 51 years ( $\mathrm{StDev}=8.2$, range 31-78years) underwent ARCR with double-loaded anchors in the 2014-15 period. The surgeon also had the availability of a knotless anchor if the repair construct dictated its use. The age of the second group of 77 patients (50 males/27 females) averaged 52 years $(\mathrm{StDev}=8.96$, range $=22-73$ years) underwent $\mathrm{ARCR}$ when generic triple-loaded anchors become available in the 2016-17 period. After the triple loaded anchor was made available, it was left to the surgeon's discretion whether to utilize a double or triple loaded anchor. The availability of a knotless anchor continued to be a choice. In all cases the anchors were molded $5.5 \mathrm{~mm}$ polyetheretherketone (PEEK) anchors loaded with ultrahigh molecular weight polyethelene (UHMWPE) sutures (RōG), a stable implant technology. A single surgeon performed all repairs on an outpatient basis.

Measures of patient's physical progress included: UCLA score, ultrasound assessment and strength recorded at 1, 3, and 6 months intervals. The RC tear pattern was recorded as the lengths in the anterior-posterior (AP) and medial-lateral (ML) dimensions in centimeters $(\mathrm{cm})$. RC tear severity was encompassed in a single metric, area $=\mathrm{AP} * \mathrm{ML}$ in $\mathrm{cm}^{2}$. The inclusion of $\mathrm{ML}$ in the metric provides a measure of the number of tendons involved in the tear, hence, tear severity.

\section{Results}

Two patient populations with similar age distributions that had arthroscopic rotator cuff repair by a single surgeon provide the basis for DL versus TL comparison. Average anchor usage as a function of RC tear size when DL and TL anchors are used is listed in Table 1. There were $48\left(<3 \mathrm{~cm}^{2}\right)$ and $21\left(\geq 3 \mathrm{~cm}^{2}\right)$ RCRs using DL anchors and 
$57\left(<3 \mathrm{~cm}^{2}\right)$ and $20\left(\geq 3 \mathrm{~cm}^{2}\right)$ using primarily TL anchors. RCTs $\geq 3 \mathrm{~cm}^{2}$ are considered large/massive tears. Average anchor use per repair when tear size is $<3 \mathrm{~cm}^{2}$ is $2.02(\mathrm{DL})$ and $1.26(\mathrm{TL})$. Average anchor use per repair when tear size is $\geq 3 \mathrm{~cm}^{2}$ is 2.95 (DL) and $1.6(\mathrm{TL})$. Use of triple-loaded anchors resulted in saving at least one anchor per RCR regardless of RCT size.

Table I Double-loaded anchor and triple-loaded anchor usage as a function of rotator cuff tear area. After triple-loaded anchors were available there were 22 repairs that also included a double loaded anchor. Knotless anchors were used in 4I RCRs before triple-loaded anchors were available and in I4 RCRs afterwards. Double and triple load anchor usage as a function of rotator cuff tear (RCT) area in $\mathrm{cm}^{2}$

\begin{tabular}{lll}
\hline $\mathbf{R C T}\left(\mathbf{c m}^{2}\right)$ & Double-load anchor average (count) & Triple-load anchor average (count) \\
\hline 1 & $1.18(\mathrm{II})$ & $1.00(9)$ \\
1.5 & $2.13(24)$ & $1.32(3 \mathrm{I})$ \\
2 & $2.33(9)$ & $1.25(16)$ \\
2.25 & $3.00(3)$ & $2.00(1)$ \\
2.5 & $3.00(1)$ & \\
3 & $2.89(10)$ & $1.17(6)$ \\
4.5 & & $2.00(1)$ \\
5 & $3.00(3)$ & $1.50(4)$ \\
6 & $2.5(2)$ & \\
7 & & $2.00(1)$ \\
8.75 & $4(1)$ & $3.00(1)$ \\
10.75 & $4(1)$ & $2.00(2)$ \\
12 & & $1.50(4)$ \\
14 & $2.50(2)$ & \\
16 & $3.00(1)$ & \\
20 & $3.00(1)$ & $2.00(1)$ \\
22.5 & & \\
\hline & & \\
\hline
\end{tabular}

The data in Table 1 are illustrated in Figure 1 as a function of tear size for double-loaded and triple-loaded anchor RCRs. Anchor usage increases from 1 to 3 anchors as tear size increases from 1 to $3 \mathrm{~cm}^{2}$ when DL-anchors are used. Anchor usage per RCR is nearly constant for tear sizes $\geq 3 \mathrm{~cm}^{2}$ : RCR DL/TL anchor average use was 2.95 (blue line) $/ 1.6$ (red line). $70 \%$ of double-load anchor repairs were for RCTs $<3 \mathrm{~cm}^{2}$ and $74 \%$ of triple-load anchor repairs were for RCTs $<3 \mathrm{~cm}^{2}$.

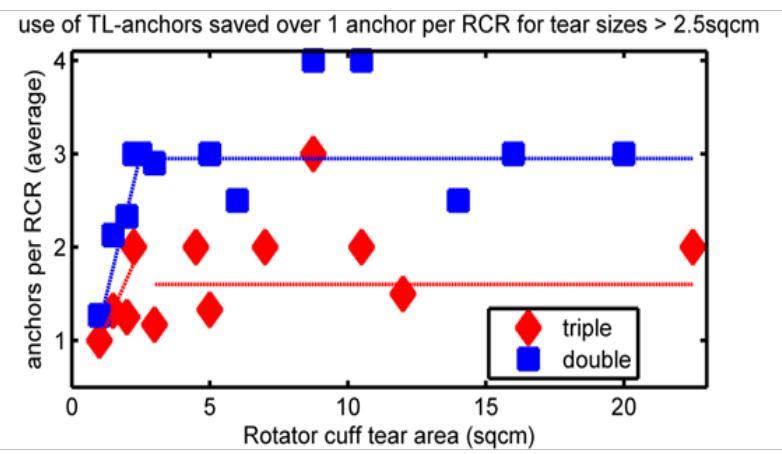

Figure I Average anchor usage as a function of rotator cuff tear area for ARCRs before and after triple-loaded anchors were used. Tear size is given in $A P^{*} M L=$ area $\left(\mathrm{cm}^{2}\right)$. RCT size in area captures tear severity better than AP length alone. Horizontal lines are averages for tear areas $\geq 3.0 \mathrm{~cm}^{2}$.

Two important points of RCR using either double or triple loaded anchors are illustrated in Table 2. First, smaller RCTs require fewer anchors while RCTs $\geq 3 \mathrm{~cm}^{2}$ repaired with double-loaded anchors required more than one additional anchor (2.95) than when tripleloaded anchors are used (1.6 anchors). Second, when all RCTs are considered (column 4), the use of triple-loaded anchors achieved greater efficiency, approximately one anchor less than when doubleloaded anchors are used (1.35 triple-loaded anchors versus 2.3 doubleloaded anchors). The reduction in the number of anchors use results in a cost savings and less compromise to the greater tuberosity footprint.

Table 2 DL:TL RCR anchor utilization

\begin{tabular}{llll}
\hline & RCTs & RCTs & RCTs \\
& $<3 \mathbf{~ s q ~ c m ~}$ & $\geq 3 \mathbf{s q ~ c m}$ & all \\
\hline patients & 57 & 20 & 77 \\
Triple-loaded anchor average & 1.26 & 1.6 & 1.35 \\
SD & 0.45 & 0.6 & 0.53 \\
patients & 48 & 21 & 69 \\
Double-loaded anchor average & 2.02 & 2.95 & 2.3 \\
SD & 0.82 & 0.63 & 0.85 \\
\hline
\end{tabular}

Comparing UCLA scores and Strength at 6 months post repair time demonstrated no difference in outcomes as a function of the use of either double-load or triple-loaded anchors (Table 3). T-test of the 
gender differences in the Summary Table resulted in insignificant two-tailed $\mathrm{P}$ value.

Table 3 Average UCLA scores for four repair types. Repeat repairs for each type are given in Column 4. UCLA scores are nearly identical for DL and TL repairs. TL Lateral combined one TL anchor and one anchor for a lateral repair. DL_TL employed one of each anchor

\begin{tabular}{llll}
\hline Repair & Ave_UCLA & Count & RCR repair \\
\hline DL & 23.9 & 22 & 0 \\
TL & 24 & 35 & 2 \\
TL_Lateral & 22.1 & 14 & 2 \\
DL_TL & 17.7 & 6 & 1 \\
\hline
\end{tabular}

\section{Discussion}

One of the few studies of the direct clinical costs of outpatient ARCR surgery an outpatient academic surgical center concluded that cost reduction should focus on reducing the number of anchors used along with anchor cost. ${ }^{12}$ It was pointed out that direct clinical costs are difficult to measure and substituting billing or charges for the cost are fraught with inherent inaccuracies. Direct costs appeared not to be related to RCT size, severity, or operative technique (e.g., single vs double row). Several factors influence total cost including BMI, ASA classification, and muscle quality along with facility utilization, pharmacy and supply costs. However, whereas many factors that affect parts of the total cost are confounders and are insignificant in the total cost. Only total number of anchors and a subscapularis repair affect total cost.

Triple-loaded anchors have been employed in an attempt to reduce the number of anchors used in RCR. ${ }^{2,13}$ In a bovine shoulder repair using TL anchors, a single-row construct was more resistant to stretching than a double-row repair. ${ }^{6}$ Another study performed on 47 patients found that DR and SR-TL repairs resulted in similar improvements in pain and function with equivalent healing rates for ARCR of $1-3 \mathrm{~cm}$ full-thickness tears. ${ }^{8} \mathrm{SR}$ repair can result in reduced operative time, complexity, and cost relative to DR repairs. There is an ongoing discussion as to whether DR has an improved proven clinical difference over the use of SR. However, DR repairs have been shown to result in better outcomes for larger RCTs as determined by structural healing. ${ }^{2,9,13-18}$ Despite multiple studies supporting the use of DR for large tears it has been suggested that cost-effectiveness research is needed to determine whether the increased implant cost and operative time for DR repairs are worth based on the outcome differences.

The present anchor usage rate is compared to anchor usage for 925 RCRs performed at the Cleveland Clinic ${ }^{11}$ performed by 13 surgeons in Figure 2. Their repairs were reported for four AP tear lengths: small $=<1 \mathrm{~cm}(\mathrm{~N}=122)$, medium $=1-3 \mathrm{~cm}(\mathrm{~N}=418)$, large $=3-5 \mathrm{~cm}(\mathrm{~N}=290)$ and massive $=>5 \mathrm{~cm}(\mathrm{~N}=95) .95 \%$ of repairs were arthroscopic using single/double row repairs. Suture anchor usage ranged from 1 to 7 , with a mean of $2.8^{ \pm} 1.32$ per case. Average anchor usage for AP length is $<3 \mathrm{~cm}$ was $2.36(\mathrm{SD}=.99)$ and $3.48(\mathrm{SD}=1.22)$ for $\mathrm{RCTs}>3 \mathrm{~cm}$. $42 \%$ of tears were $>3 \mathrm{~cm}$. These results are compared to TL-anchor average use in this study in Figure 2. When AP tear length is $<3 \mathrm{~cm}$ there appears to be no difference in anchor usage. When AP length is $\geq 3 \mathrm{~cm}$ there is a savings in anchor use per case of approximately one anchor per case.

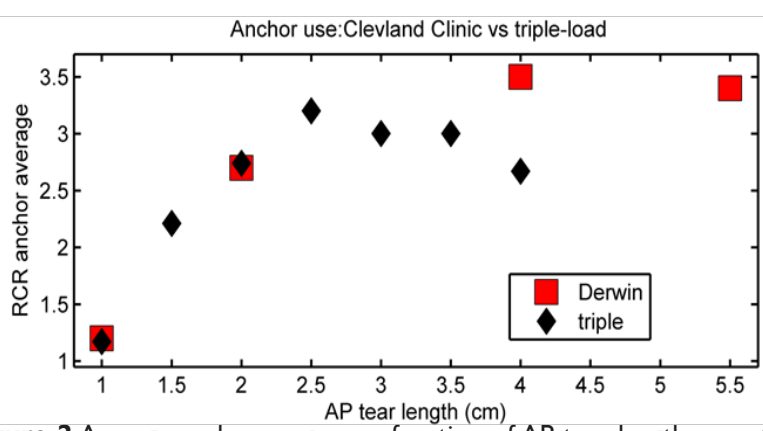

Figure 2 Average anchor usage as a function of AP tear length reported by Derwin et al." compared to the TL-anchor use reported here.

\section{Conclusion}

The use of triple- loaded anchors can result in significant savings for repair of large RC tears defined as either AP length $>3 \mathrm{~cm}$ or tear area $\geq 3 \mathrm{~cm}^{2}$. The use of AP*LM as a measure of tear size is more descriptive than AP length as it encompasses the number of tendons involved in the tear. Cost savings result from the elimination of one anchor per case for large tears.

\section{Acknowledgments}

None.

\section{Conflicts of interest}

The authors declare there is no conflict of interest.

\section{References}

1. Colvin AC, Egorova N, Harrison AK, et al. National trends in rotator cuff repair. J Bone Joint Surg. 2012;94(3):227-233.

2. Mascarenhas R, Chalmers PN, Sayegh ET, et al. Is double-row rotator cuff repair clinically superior to single-row Rotator cuff repair: A systematic review of overlapping meta-analysis. Arthroscopy. 2014;30(9):11561165 .

3. Hohmann E, König A, Kat CJ, et al. Single- versus double-row repair for full-thickness rotator cuff tears using suture anchors. A systematic review and meta-analysis of basic biomechanical studies. Eur J Orthop Surg Traum. 2018;28(5):859-868.

4. Aydin N, Karaismailoglu B, Gurcan M, et al. Arthroscopic double-row rotator cuff repair: a comprehensive review of the literature. SICOT-J. 2018;4:1-10.

5. Castagna A, Garofalo R, Conti M, et al. Arthroscopic rotator cuff repair using a triple-loaded suture anchor and a modified Mason-Allen technique (Alex stitch). Arthroscopy. 2007;23(4):440-444.

6. Barber FA, Herbert MA, Schroeder A, et al. Biomechanical advantages of triple-loaded suture anchors compared with double-row rotator cuff repair. Arthroscopy. 2010;26(3):316-323.

7. Noyes MP, Lederman E, Adams CR, et al. Triple-loaded suture anchors versus a knotless rip stop construct in a single-row rotator cuff repair model. Arthroscopy. 2018;34(5):1414-1420.

8. Tashjian RJ, Granger EK, Chalmers PN. Healing rates and functional outcomes after triple-loaded single-row versus transosseous-equivalent double-row rotator cuff tendon repair. Orthop J Sports Med. 2018;6(11):17.

9. Lorbach O, Kieb M, Raber F, et al. Comparable biomechanical results for a modified single-row rotator cuff reconstruction using triple-loaded suture anchors versus a suture-bridging double-row repair. Arthroscopy. 2012;28(2):178-187. 
10. Rhode BA, Rhode WS. Cost comparison of rotator cuff repair between double and triple loaded anchor. MOJ Orthopedics \& Rheumatology. 2016;4(6):1-4

11. Derwin KA, Sahoo S, Zajichek A, et al. Tear characteristics and surgeon influence repair technique and suture anchor use in repair of superior-posterior rotator cuff tendon tears. J Shoulder Elbow Surg. 2019;28(2):227-236.

12. Tashjian RZ, Belisle J, Baran S, et al. Factors influencing direct clinical costs of outpatient arthroscopic rotator cuff repair surgery. J Shoulder Elbow Surg. 2018;27(2):237-241.

13. Mascarenhas R, Khair M, Verma NN, et al. Single-row rotator cuff repair: Speedfix and triple-loaded anchor. Tech Shoul Elbow Surg. 2017;18(4):121-128.

14. Gartsman GM, Drake G, Edwards TB, et al. Ultrasound evaluation of arthroscopic full-thickness supraspinatus rotator cuff repair: single-row versus double-row suture bridge (transosseous equivalent) fixation. Results of a prospective, randomized study. J Shoulder Elbow Surg. 2013;22(11):1480-1487.
15. Carbonel I, Martinez AA, Calvo A, et al. Single-row versus double-row arthroscopic repair in the treatment of rotator cuff tears: a prospective randomized clinical study. Int Orthop. 2012;36(9):1877-1883.

16. Park JY, Lhee SH, Choi JH, et al. Comparison of the clinical outcomes of single- and double-row repairs in rotator cuff tears. Am J Sports Med. 2008;36(7):1310-1316.

17. Ma CB, Comerford L, Wilson J, et al. Biomechanical evaluation of arthroscopic rotator cuff repairs: double-row compared with single-row fixation. J Bone Joint Surg Am. 2006;88(2):403-410.

18. Lapner PL, Sabri E, Rakhra K, et al. A multicenter randomized controlled trial comparing single-row with double-row fixation in arthroscopic rotator cuff repair. J Bone Joint Surg Am. 2012;94(14):1249-1257. 